COLBY-93-05

IUHET 256

\title{
ATOMIC SUPERSYMMETRY, RYDBERG WAVE PACKETS, AND RADIAL SQUEEZED STATES
}

\author{
Robert Bluhm ${ }^{a}$ and V. Alan Kostelecký ${ }^{b}$ \\ ${ }^{a}$ Physics Department \\ Colby College \\ Waterville, ME 04901, U.S.A \\ ${ }^{b}$ Physics Department \\ Indiana University \\ Bloomington, IN 47405, U.S.A.
}

\begin{abstract}
We study radial wave packets produced by short-pulsed laser fields acting on Rydberg atoms, using analytical tools from supersymmetrybased quantum-defect theory. We begin with a time-dependent perturbative calculation for alkali-metal atoms, incorporating the atomicexcitation process. This provides insight into the general wave packet behavior and demonstrates agreement with conventional theory. We then obtain an alternative analytical description of a radial wave packet as a member of a particular family of squeezed states, which we call radial squeezed states. By construction, these have close to minimum uncertainty in the radial coordinates during the first pass through the outer apsidal point. The properties of radial squeezed states are investigated, and they are shown to provide a description of certain aspects of Rydberg atoms excited by short-pulsed laser fields. We derive expressions for the time evolution and the autocorrelation of the radial squeezed states, and we study numerically and analytically their behavior in several alkali-metal atoms. Full and fractional revivals are observed. Comparisons show agreement with other theoretical results and with experiment.
\end{abstract}

Published in Physical Review A 49, 4628 (1994) 


\section{INTRODUCTION}

When an atom is excited from its ground state by a short-pulsed laser field, a localized radial wave packet is produced that has behavior mimicking the classical radial motion of a charged particle in a Coulomb field [1, 2, 3, 泪. The properties and time evolution of such wave packets provide interesting experimental and theoretical opportunities to probe the interface between classical and quantum mechanics.

Experiments have detected the periodic motion of the electron in a Rydberg atom excited by a short laser pulse, with period equal to the classical period of a particle in a keplerian orbit [5, 6]. The motion of the wave packet is only partly classical, however. For excitations by a single laser pulse from the atomic ground state, the angular distribution is that of a p state. Also, the radial wave packet disperses over the course of time. However, after many Kepler times, the wave packet remnants eventually recombine into a packet that is close to the original shape and that oscillates radially with the keplerian orbital period. This recombined wave packet is called a full revival. In the intervals between the initial and the fully revived classical motions, subsidiary wave packets form. These are called fractional revivals, and they have orbital periods equal to rational fractions of the classical keplerian period. Both full and fractional revivals have been seen experimentally [7, 8, 9], and descriptions of their behavior have been developed [10, 11, 12].

Several theoretical approaches have been used to study the properties of radial wave packets formed by excitation of Rydberg atoms with short laser pulses. Refs. [1, 2] solve the Schrödinger equation numerically, while for gaussian shaped laser pulses and weak-field excitations, Refs. [3, [] use perturbation theory. For a particular class of pulse shapes, Ref. [13] is able to obtain nonperturbative solutions. Also,

Ref. [14] provides a theoretical description for the generation of Ramsey fringes with Rydberg wave packets.

Since the radial wave packets are initially localized in the radial coordinates and partly follow the classical motion, a description in terms of some kind of coherent state [15] might seem appropriate. However, standard coherent-state approaches to the hydrogenic Coulomb problem [16, 17, 18, 19, 20] and to short-pulse laser excitation 
of Rydberg atoms 21, 22, 23 have yielded descriptions of the motion in a Coulomb potential with the electron moving on circular or elliptical orbits. None of these match the behavior of Rydberg atoms in short-pulsed laser fields with p-state angular distributions. Within this framework, the issue of an analytical construction of radial wave packets for Rydberg atoms prepared by excitation with short laser pulses with no external fields present has been an open problem.

Recently, we have provided a framework for an analytical study of Rydberg wave packets [24] and have discussed some of their properties for hydrogen. In the present work, we generalize this method to non-hydrogenic atoms, with particular emphasis on the alkali-metal atoms for which experiments have been performed. We also extend our analysis and obtain further results for the hydrogenic case.

To incorporate non-hydrogenic features in the treatment, we address matters in the context of supersymmetry-based quantum-defect theory (SQDT) [25, 26]. This theory describes the behavior of an excited Rydberg electron as that of a single particle in an effective central potential. The effective potential can be found by acting on the hydrogenic Coulomb potential with a symmetry transformation and adding a symmetry-breaking term. The symmetry transformation used is a quantummechanical supersymmetry [27]. The supersymmetry-breaking term incorporates nonhydrogenic contributions to the effective potential. Although the model is analytical, its combination of supersymmetry ideas with the notion of quantum defects [28, 29, 30, 31] is such that exact energy eigenvalues are generated. The corresponding analytical eigenfunctions can be used to provide predictions for transition probablilities of alkali-metal atoms [26] and alkaline-earth-metal ions [32], in good agreement with accepted values. The model also can be used to generate Stark maps for the alkali-metal atoms [33] that agree with experiment and standard theory [34, 35]. The validity of the model breaks down inside the electronic core, although some bulk features of the fine structure of alkali-metal atoms are reproduced [36]. Extensions of these ideas apply to other situations including, for example, the Penning trap [37. For recent overviews of atomic supersymmetry, see Ref. [38].

The analytical inclusion of quantum defects for Rydberg atoms suggests that SQDT could be a useful tool for the study of Rydberg atoms in short-pulsed laser 
fields. We have identified two approaches as being especially promising in this regard. One is the inclusion of SQDT in conventional time-dependent perturbation theory to explore the formation and behavior of radial wave packets, in particular to show the presence of oscillatory motion between the orbital apsidal points. The results can then be compared with those obtained using numerical approaches [4], 2] and WKB solutions in perturbation theory [3, 国].

The second approach we adopt is the use of SQDT in the construction of a family of analytical squeezed states, which we call radial squeezed states (RSS), that can represent radial wave packets formed in a Rydberg atom excited by a short laser pulse. For our purposes, given a hamiltonian and a commutation relation, a squeezed state can be taken as a wave function that satisfies the corresponding minimum-uncertainty relation at a particular time but with uncertainties not necessarily those of the ground state. For the simple harmonic oscillator, for example, the uncertainty product of a squeezed state has a sinusoidal dependence on time, with the uncertainties in coordinate and momentum space oscillating while the center of the wave packet follows the classical motion 39.

The analytical method we use to obtain the RSS is discussed in Sec. IV. The result is a three-parameter family of wave packets with the angular dependence of a $p$ state. For an individual packet, the values of the three parameters are fixed in terms of expectations of the energy, momentum, and position when the wave packet first reaches the outer apsidal point. This initialization condition agrees both with the results of other theoretical calculations and with the intuitive notion that the electron wave packet attains closest-to-classical behavior when localized at large quantum numbers immediately after its formation. The ensuing behavior is completely determined by the time-dependent Schrödinger equation.

Since the RSS are given by a relatively simple analytical expression, their time evolution can be studied either analytically or numerically. We have used both approaches. Quantities of interest are the time dependence of the RSS and of autocorrelation functions with the initial packet. In what follows, we examine these for several different alkali-metal atoms. We treat issues such as the variation with the quantum defect of the orbital period and of the full or fractional revivals. 
This paper is organized as follows. In the next section, we summarize some necessary background information on the salient features of SQDT and on the various approaches to the study of radial wave packets formed with short-pulsed laser fields applied to Rydberg atoms. In Sec. III, we use SQDT to perform a time-dependent perturbative calculation for the formation and time evolution of the radial wave packets. The derivation and basic properties of the RSS are described in Sec. IV. Section $\mathrm{V}$ discusses their time-dependent properties, including their time evolution and their autocorrelation functions, comparing with other available theoretical results and with some experiments. A summary and our conclusions are given in Sec. VI. Throughout this work, we use atomic units with $\hbar=e=m_{e}=1$.

\section{BACKGROUND}

In this section, we provide background material on atomic supersymmetry and on prior theoretical treatments of Rydberg atoms in short-pulsed laser fields.

\section{A. Atomic Supersymmetry}

Atomic supersymmetry involves a particular realization $\mathcal{R}$ of the superalgebra $\operatorname{sqm}(2)$. The three generators of $\operatorname{sqm}(2)$ can be taken as the hamiltonian $H_{s s}$ of the supersymmetric quantum system, the supersymmetry charge $Q$, and its conjugate $Q^{\dagger}$. They obey the relations

$$
\left[H_{s s}, Q\right]=\left[H_{s s}, Q^{\dagger}\right]=0 \quad, \quad\left\{Q, Q^{\dagger}\right\}=H_{s s}
$$

In the realization $\mathcal{R}$, the hamiltonian for $\operatorname{sqm}(2)$ splits as the direct $\operatorname{sum} H_{s s}=$ $H_{+} \oplus H_{-}$. The component hamiltonians $H_{+}$and $H_{-}$are one-variable differential operators satisfying the eigenequations

$$
H_{ \pm} \Psi_{ \pm n} \equiv\left[-\frac{d^{2}}{d x^{2}}+V_{ \pm}(x)\right] \Psi_{ \pm n}=\epsilon_{n} \Psi_{ \pm n}
$$

where the potentials $V_{+}$and $V_{-}$are supersymmetric partners conventionally expressed in terms of $x$-derivatives of a function $U(x)$ :

$$
V_{ \pm}(x)=\left(\frac{1}{2} U^{\prime}\right)^{2} \mp \frac{1}{2} U^{\prime \prime}
$$


The ground-state eigenvalue is zero and is associated with $H_{+}$only. The spectra of the two hamiltonians are otherwise identical [27.

When the hamiltonian $H_{+}$is identified with the differential operator for the radial part of the hydrogen-atom Schrödinger equation expressed in spherical polar coordinates, the supersymmetry partner $H_{-}$can be constructed 25] by fixing $l$ and solving for $V_{-l}$ in Eq. (2). It can then be shown that the supersymmetric eigenfunction partners are $R_{n l}$ with $n \geq 1$ and $R_{n, l+1}$ with $n \geq 2$. A physical interpretation of these results has been proposed [25], leading to sets of nested supersymmetries interconnecting eigenvalues and eigenstates of different atoms and ions. For example, in the exact symmetry limit where electron-electron interactions can be neglected, the s levels of lithium may be regarded as the supersymmetric partners of the hydrogen atom s levels. The s orbitals of sodium can then in turn be viewed as supersymmetric partners of the lithium s orbitals. Connections also exist among p and higher orbitals. An integer shift in the angular quantum number $l$ accompanies each supersymmetry operation.

In real atoms such as alkali metals, the exact supersymmetry is broken by coupling between the valence electron and the electronic core. One result is a shift in eigenenergies [28], $E_{n} \rightarrow E_{n^{*}}=-1 / 2 n^{* 2}$, relative to hydrogenic values. The quantity $n^{*}=n-\delta(n, l)$ incorporates these shifts via quantum defects $\delta(n, l)$. As $n$ increases, the exact quantum defects rapidly approach asymptotic values $\delta(l)$.

The basic idea of SQDT is to incorporate these shifts in an analytical one-particle hamiltonian [26]. The model is obtained by adding to the exact supersymmetric hamiltonian specific supersymmetry-breaking terms, chosen so that the quantumdefect eigenenergies are reproduced while leaving the eigenfunctions analytical. A modified angular quantum number $l^{*}=l-\delta(l)+I(l)$ is introduced, where $I(l)$ is an integer playing the role of the supersymmetric shift. The SQDT hamiltonian is found via the replacement $n, l, E_{n} \rightarrow n^{*}, l^{*}, E_{n^{*}}$ in the radial Coulomb equation. The corresponding SQDT eigenfunctions are given by

$$
R_{n^{*} l^{*}}(r)=\frac{2}{n^{* 2}}\left[\frac{\Gamma\left(n^{*}-l^{*}\right)}{\Gamma\left(n^{*}+l^{*}+1\right)}\right]^{\frac{1}{2}}\left(\frac{2 r}{n^{*}}\right)^{l^{*}} \exp \left(-\frac{r}{n^{*}}\right) L_{n^{*}-l^{*}-1}^{\left(2 *^{*}+1\right)}\left(\frac{2 r}{n^{*}}\right)
$$

The full three-dimensional SQDT wave functions are therefore $R_{n^{*} l^{*}}(r) Y_{l m}(\theta, \phi)$. For 
asymptotic quantum defects $\delta(l)$, these eigenfunctions form a complete and orthonormal set. Note that in the limit of vanishing quantum defects $\delta(l)$ and supersymmetry integers $I(l)$ the usual Coulomb eigenvalues and eigenfunctions are recovered.

\section{B. Radial Wave Packets}

The time-dependent Schrödinger equation for a hydrogen atom in a laser field is

$$
i \frac{\partial}{\partial t} \Psi(t)=\left(H_{0}-\vec{\mu} \cdot \vec{E}\right) \Psi(t)
$$

where $H_{0}$ is the zero-field hamiltonian, $\vec{\mu}$ is the atomic dipole moment, and $\vec{E}$ is the laser field. Consider an atom excited by a single photon from an initial state $|i\rangle$ to a range of Rydberg states by a laser pulse of length $\tau$ and frequency $\omega$. The electric field can be written as

$$
\vec{E}(t)=\mathcal{E}(t) \vec{\epsilon} e^{-i \omega t}+\text { c.c. } .
$$

Here, $\vec{\epsilon}$ is the polarization vector and $\mathcal{E}(t)$ is a gaussian envelope for the laser pulse,

$$
\mathcal{E}(t)=\mathcal{E}_{0} \exp \left(-\frac{4(\ln 2) t^{2}}{\tau^{2}}\right)
$$

which is taken to be centered at $t=0$ with full width at half maximum equal to $\tau$.

If we expand $\Psi(t)$ in terms of hydrogenic wave functions,

$$
\Psi(t)=\sum_{n l m} a_{n l m}(t) e^{-i E_{n} t} R_{n l} Y_{l m}
$$

and use the rotating wave approximation, we obtain equations for the time-dependent parameters $a_{n l m}(t)$. These equations may be solved numerically [1, 2] or perturbatively for weak fields [3, 国. Quantities of interest, such as the probability distribution $r^{2}|\Psi(r, t)|^{2}$ of the radial part of the wave function, may then be plotted as a function of time. Refs. [1, 2, 3, [4] use hydrogenic dipole moments and energy spacings for simplicity, although numerical methods incorporating standard quantum-defect theory can be applied. Refs. [6, 0, 8] contain numerical calculations for alkali-metal atoms.

The results obtained by these procedures show that a radial wave packet forms shortly after the initial laser excitation. The packet subsequently oscillates between the radial apsidal points. Refs. [1, 2, 3, (4) consider an 8 to 10 psec laser pulse 
that excites a range of $\mathrm{p}$ states of hydrogen centered about the value $\bar{n}=85$ of $n$. The resulting wave packet is calculated to oscillate with a period equal to the classical Kepler period $T_{\mathrm{cl}}=2 \pi \bar{n}^{3} \simeq 93.3$ psec. At the inner turning point, near the origin, the distribution $r^{2}|\Psi(r, t)|^{2}$ of the packet is broad and exhibits many oscillations. As the packet returns to the outer turning point, near $2 \bar{n}^{2}=14450$ a.u., the distribution becomes narrower and increases in amplitude, while the number of oscillations decreases significantly. At the first pass through the outer turning point, the wave packet is calculated to have close to minimum uncertainty in the radial coordinates [1, 2].

\section{TIME-DEPENDENT PERTURBATION THEORY}

In this section, we show how to perform a time-dependent perturbative calculation that incorporates SQDT into the analysis. The calculation describes the formation and time evolution of a Rydberg atom excited from its ground state by a short laser pulse. The field is taken to have the form in Eq. (6) with envelope $\mathcal{E}(t)$ as in Eq. (7).

We begin by expanding the wave function $\Psi(t)$ describing alkali-metal Rydberg states in terms of the complete and orthonormal set of SQDT states $R_{n^{*} l^{*}}(r) Y_{l m}(\theta, \phi)$. For single photon excitations from the ground state, only p-state angular wave functions are needed. However, the procedure that follows can be generalized to multiphoton processes that excite $\mathrm{d}$ or higher states. We neglect any contributions from the continuum, which are negligible for the cases of interest. The expansion (8) therefore becomes

$$
\Psi(t)=Y_{10}(\theta, \phi) \sum_{n} a_{n}(t) e^{-i E_{n^{*} t}} R_{n^{*} l^{*}}(r)
$$

where now $n^{*}=n-\delta(1)$ and $l^{*}=1-\delta(1)+I(1)$, with $\delta(1)$ the p-state quantum defect and $I(1)$ the p-state supersymmetry integer. We then use the rotating wave approximation and solve for $a_{n}(t)$ to lowest order in perturbation theory. For times $t \gg 0$, we get

$$
a_{n}=i\left\langle n^{*}, l^{*}|\vec{\mu} \cdot \vec{\epsilon}| i\right\rangle \tilde{\mathcal{E}}\left(\delta_{n}\right),
$$

where $|i\rangle$ denotes the ground state and $\tilde{\mathcal{E}}\left(\delta_{n}\right)$ is the Fourier transform of $\mathcal{E}(t)$ with $\delta_{n}=\left(E_{n^{*}}-E_{i^{*}}-\omega\right)$. Note that the eigenenergies $E_{n^{*}}=-1 / 2 n^{* 2}$, including the 
ground-state energy $E_{i^{*}}$, incorporate the quantum defect.

The dipole matrix elements $\left\langle n^{*}, l^{*}|\vec{\mu} \cdot \vec{\epsilon}| i\right\rangle$ can be evaluated analytically for different alkali-metal atoms [26]. Equation (4) specifies the form of the radial wave functions $R_{n^{*} l^{*}}(r)$. Then, for fixed $\theta$ and up to a normalization constant $N$, the radial probability density is given by

$$
r^{2}|\Psi(t)|^{2}=N r^{2}\left|\sum_{n} R_{n^{*} l^{*}}(r)\left\langle n^{*}, l^{*}|r| 1,0\right\rangle \exp \left(-\frac{\left(E_{n^{*}}-E_{\bar{n}^{*}}\right)^{2} \tau^{2}}{16 \ln 2}\right) e^{-i E_{n^{*} t}}\right|^{2}
$$

In this expression, we have taken the laser frequency $\omega$ as tuned to excite a range of energies centered on $E_{\bar{n}^{*}}$,

$$
\omega=E_{\bar{n}^{*}}-E_{i^{*}}
$$

Equation (11) holds for $t \gg 0$. We can modify the procedure and evaluate $r^{2}|\Psi(t)|^{2}$ for $t=0$ as well. The resulting calculation shows that the answer can be written by making the substitution

$$
e^{-i E_{n^{*} t}} \rightarrow \frac{1}{2}\left[1+\operatorname{erf}\left(-i \sqrt{\frac{\left(E_{n^{*}}-E_{\bar{n}^{*}}\right)^{2} \tau^{2}}{16 \ln 2}}\right)\right]
$$

in Eq. (11), where erf(z) is the error function.

As a first example, consider hydrogen. This element has $\delta(1)=I(1)=0$, so $n^{*}=n$. For purposes of comparison with Ref. [3], we take $\bar{n}^{*}=\bar{n}=85$ and $\tau=8$ psec. Figure 1 displays the radial probability distribution $f(r)=r^{2}|\Psi(t)|^{2}$ at several different times. From left to right, these are $t=\frac{1}{9} T_{\mathrm{cl}} \simeq 10.3 \mathrm{psec}, \frac{2}{9} T_{\mathrm{cl}} \simeq 20.7 \mathrm{psec}$, $\frac{3}{9} T_{\mathrm{cl}} \simeq 31.1 \mathrm{psec}$, and $\frac{4}{9} T_{\mathrm{cl}} \simeq 41.5$ psec, where $T_{\mathrm{cl}}=2 \pi \bar{n}^{3} \simeq 93.3$ psec is the classical orbital period. No vertical scale is shown because the packet is unnormalized. The figure uses Eq. (11) evaluated numerically over the subset of states $n=75-95$, which we determined to be sufficient for high accuracy.

The graph reveals that during formation the radial wave packet moves away from the origin, increasing in amplitude as it approaches the outer turning point $r_{\mathrm{cl}} \approx 2 n^{2}=$ 14450 a.u. It then decreases in amplitude as it starts to bounce back towards the origin. Our Fig. 1 agrees with Figure 1 of Ref. [3] in its gross features, although there are differences in the detailed shapes of the packets. Ref. [3] uses a WKB solution to evaluate the Coulomb functions, whereas we use Eq. (11) directly and evaluate 
the associated Laguerre polynomials in $R_{n^{*} l^{*}}(r)$ with high numerical accuracy for a subset of states.

As a second example, we look at rubidium. This has $\delta(1) \simeq 2.65$ and $I(1)=3$. For purposes of comparison with the hydrogenic case, we again choose $\tau=8$ psec and $\bar{n}=85$. This corresponds to $\bar{n}^{*} \simeq 82.35$. As we show in Sec. IV, the inequivalence of $\bar{n}$ and $\bar{n}^{*}$ means that the classical orbital period is reduced from the hydrogenic value to $T_{\mathrm{cl}}^{*}=2 \pi \bar{n}^{* 3} \simeq 84.9$ psec. Figure 2 displays the radial probability distribution $f(r)$ for rubidium at the same times as in Fig. 1. The overall behavior is similar to that of hydrogen. However, comparison of Figs. 1 and 2 shows that the outer turning point $r_{\mathrm{cl}}^{*}$ for rubidium is slightly smaller than for hydrogen. This difference is due to the quantum defects. In fact, as we show in Sec. IV A, the outer turning point is $r_{\mathrm{cl}}^{*} \approx 2 \bar{n}^{* 2} \simeq 13560$ a.u. here.

\section{RADIAL ATOMIC SQUEEZED STATES}

The results in the previous section demonstrate that the analytical framework provided by SQDT is suitable for the perturbative study of the time evolution of radial wave packets for different alkali-metal atoms. However, the development and application of an analytical treatment of the problem that avoids perturbation theory is evidently of interest and is the principal goal of this paper.

The uncertainty product $\Delta r \Delta p_{r}$ is close to its minimum value during the first passage of the wave packet through the outer turning point [1], 2]. This suggests that we seek an analytical description of a radial wave packet at its outer turning point as some type of minimum-uncertainty wave function.

In this section, we use SQDT to derive a family of analytical wave packets that obey minimum-uncertainty relations for certain special variables and that have uncertainty product $\Delta r \Delta p_{r}$ close to the minimum value. We call these packets radial squeezed states (RSS). The use of SQDT ensures that the RSS are relevant for all alkali-metal atoms, not merely hydrogen, and permits us to take into account the quantum defects of the different alkali-metal atoms used in experiments. Discussion 
of the time evolution of the RSS is deferred to Sec. V.

\section{A. Classical Motion}

The construction of close-to-classical (approximate minimum-uncertainty) wave functions is aided by an understanding of classical behavior. In this subsection, we consider the motion of a classical particle in a central potential that can be viewed as the classical limit of the effective potential in SQDT.

In general, the motion of a particle in a central potential $U(r)$ is governed by the lagrangian

$$
L=\frac{1}{2} \dot{r}^{2}+\frac{1}{2} r^{2} \dot{\theta}^{2}-U(r)
$$

The classical angular momentum $l=r^{2} \dot{\theta}$ is constant and so can be used to reduce the equations of motion to a single equation for the radial coordinate $r$.

The SQDT uses an $l$-dependent effective central potential $U(r)$. One perspective is therefore that it replaces the intractable many-body action for the multi-electron atom with an infinite set of single-particle effective actions, labeled by $l$. Each member of this set describes the behavior of the system for the given value of the angular momentum. In the classical case, $l$ is a continuous variable, and the central potential $U(r)$ can be taken as

$$
U(r)=-\frac{1}{r}+\frac{l^{* 2}-l^{2}}{2 r^{2}}
$$

This potential generates an effective radial hamiltonian

$$
H^{*} \equiv \frac{1}{2} p_{r}^{2}+\frac{l^{* 2}}{2 r^{2}}-\frac{1}{r}=E^{*}
$$

where $p_{r}=\dot{r}$ is the radial momentum and $E^{*}$ is the energy. Note that when $l^{*}=l$, the classical SQDT reduces to the usual Coulomb case.

For negative $E^{*}$, the radial motion is oscillatory between two values $r_{1,2}$ of $r$ corresponding to the apsidal points of the orbit and given by

$$
r_{1,2}=\frac{1}{2\left|E^{*}\right|}\left(1 \pm \sqrt{1-2\left|E^{*}\right| l^{* 2}}\right)
$$

The classical orbital period $T_{\mathrm{cl}}^{*}$ for the SQDT can be defined as the time taken to 
move from $r_{1}$ to $r_{2}$ and back. Using Eq. (16), a short calculation gives

$$
T_{\mathrm{cl}}^{*}=\frac{\pi}{\sqrt{2\left|E^{*}\right|^{3}}} .
$$

Bertrand's theorem implies that for the SQDT central potential (15) the orbits are not closed. However, the classical equations of motion can be solved exactly, as might be expected from the analytical nature of the quantum theory. The solution for the classical orbit can be found by eliminating $t$ from Eq. (16), using $d \theta=l d t / r^{2}$, and integrating. We thereby obtain the orbit equation

$$
\frac{1}{r}=\frac{1}{l^{* 2}}\left(1+e \cos \left[f\left(\theta-\theta_{0}\right)\right]\right) \quad .
$$

Here, $\theta_{0}$ is a constant of the integration, and we have defined

$$
f=\frac{l^{*}}{l}
$$

and

$$
e=\sqrt{1-2\left|E^{*}\right| l^{* 2}} .
$$

Equation (19) describes a precessing ellipse, with semimajor axis $a=1 / 2\left|E^{*}\right|$ and eccentricity $e$. The apsidal points of the orbit are $r_{1,2}=a(1 \pm e)$. The precession in one orbital period is $\Delta \theta=2 \pi(1-1 / f)$. Evidently, the precession is a classical consequence of the presence in the SQDT of the supersymmetry-type shifts and the quantum defects. Note that for different atomic levels the quantity $f$ in the SQDT can be larger or smaller than one, so the precession may be clockwise or counterclockwise. Also, if $l^{*}=l$ then $f=1$ and the precession disappears.

For convenience in comparing with the quantum theory, it is useful to write $E^{*}$ as

$$
E^{*}=-\frac{1}{2 n^{* 2}} .
$$

The choice of notation reflects that in the quantum theory $n^{*}$ is the quantized, shifted principal quantum number. However, at the classical level, it is a convenient, continuous variable. The classical turning points and the classical orbital period can be expressed in terms of $n^{*}$ as

$$
r_{1,2}=n^{* 2}\left(1 \pm \sqrt{1-\frac{l^{* 2}}{n^{* 2}}}\right)
$$


and

$$
T_{\mathrm{cl}}^{*}=2 \pi n^{* 3}
$$

The orbital eccentricity becomes

$$
e=\sqrt{1-\frac{l^{* 2}}{n^{* 2}}}
$$

For single-photon excitation of a Rydberg atom to a wave packet with large average principal quantum number, the ratio $l^{*} / n^{*}$ is small. This means that the RSS we derive below and the radial wave packets experimentally constructed have corresponding classical orbits that are highly elliptical, with $e \simeq 1$.

\section{B. Oscillator Description of Radial Motion}

We have found that direct attempts to construct minimum-uncertainty wave functions for the SQDT meet intractable difficulties. In fact, the same is true of the pure hydrogenic case, as has been known since the time of Schrödinger [16]. We sidestep the issue by replacing $r$ and $p_{r}$ with a new set of classical variables, $R$ and $P$, in terms

of which the description of the motion is close to that of a harmonic oscillator. The new radial variable $R$ is chosen to have a simple sinusoidal dependence on the angle $\theta$. The resulting equations are relatively easy to handle at the quantum level, so that minimum-uncertainty packets can be found analytically. This elegant technique was originally introduced in Ref. [19] to construct 'minimum-uncertainty coherent states' for the Coulomb problem, which correspond in the present context to states with high angular momentum (and small eccentricity).

For simplicity, we pick $\theta_{0}$ such that $f \theta_{0}=\frac{\pi}{2}$. The new radial variable is then given as

$$
R \equiv \frac{1}{r}-\frac{1}{l^{* 2}}=\frac{e}{l^{* 2}} \sin f \theta
$$

Its time derivative is

$$
\dot{R}=\frac{e}{l^{*} r^{2}} \cos f \theta
$$

and the corresponding conjugate momentum $P$ is

$$
P \equiv-\frac{r^{2}}{f} \dot{R}=-\frac{e l}{l^{* 2}} \cos f \theta
$$


The classical equations of motion can be written

$$
\begin{gathered}
\dot{R}=-\frac{f}{r^{2}} P, \\
\dot{P}=\frac{l^{2} f}{r^{2}} R .
\end{gathered}
$$

The new classical variables satisfy the relations

$$
\begin{gathered}
\partial_{r} R=\frac{\dot{R}}{\dot{r}}=\frac{1}{l^{*} r} \sqrt{\frac{e^{2}-l^{* 4} R^{2}}{2 E r^{2}+2 r-l^{* 2}}}, \\
P=\frac{1}{f} \dot{r}=\frac{p_{r}}{f} .
\end{gathered}
$$

These can be used to reexpress Eq. (16) as

$$
\frac{1}{2} P^{2}+\frac{1}{2} l^{2} R^{2}=\frac{e^{2}}{2 f^{2}}
$$

This equation has the form of the energy equation for a simple harmonic oscillator of frequency $l$ and energy $e^{2} / 2 f^{2}$.

\section{Derivation of Radial Squeezed States}

We next pass to the quantum level. The quantum radial hamiltonian for SQDT is

$$
H=-\frac{1}{2}\left(\frac{d^{2}}{d r^{2}}+\frac{2}{r} \frac{d}{d r}\right)-\frac{1}{r}+\frac{l^{*}\left(l^{*}+1\right)}{2 r^{2}} .
$$

The canonical radial coordinate $r$ and its conjugate momentum $p_{r}=-i\left(\partial_{r}+\frac{1}{r}\right)$ obey the commutation relation $\left[r, p_{r}\right]=i$. The eigensolutions for this hamiltonian are given in Eq. (4).

The quantum operators corresponding to the new classical variables $R, P$ are

$$
\begin{gathered}
R=\frac{1}{r}-\frac{1}{l^{*}\left(l^{*}+1\right)}, \\
P=\frac{p_{r}}{f}=-\frac{i}{f}\left(\partial_{r}+\frac{1}{r}\right) .
\end{gathered}
$$

They obey the commutation relation

$$
[R, P]=-\frac{i}{f} \frac{1}{r^{2}}
$$


The presence of the factor $1 / r^{2}$ in this equation reflects the unconventional choice of coordinate $R$ and shows that, despite the similarities described above, the system is not a true quantum-mechanical simple harmonic oscillator.

The uncertainty product $\Delta R \Delta P$ follows from Eq. (37) and is given by

$$
\Delta R \Delta P \geq \frac{1}{2 f}\left\langle\frac{1}{r^{2}}\right\rangle
$$

At a fixed time, the minimum-uncertainty wave functions therefore satisfy

$$
(R-\langle R\rangle) \psi=i A(P-\langle P\rangle) \psi
$$

where

$$
A=\frac{2 f(\Delta R)^{2}}{\left\langle\frac{1}{r^{2}}\right\rangle}=\frac{\Delta R}{\Delta P}
$$

is a real constant. Defining the parameters

$$
\alpha=\frac{f}{A}-1, \quad \gamma_{0}=\frac{f}{A}\left\langle\frac{1}{r}\right\rangle, \quad \gamma_{1}=-\left\langle p_{r}\right\rangle
$$

equation (39) becomes

$$
\left(\partial_{r}-\frac{\alpha}{r}+\left(\gamma_{0}+i \gamma_{1}\right)\right) \psi(r)=0
$$

The solution to Eq. (42) is

$$
\psi(r)=N r^{\alpha} e^{-\gamma_{0} r} e^{-i \gamma_{1} r}
$$

where $N$ is a normalization constant. These states form a three-parameter family of squeezed states minimizing the uncertainty relation (38) at a fixed time. These are our radial squeezed states (RSS).

Our derivation has demonstrated that, in any atom for which SQDT provides a good description, the RSS are candidates for Rydberg wave packets. Moreover, they have a relatively simple analytical form. In particular, these results apply to the alkali-metal atoms rubidium and potassium, for which the experiments on radial wave packets have been done to date.

For the special choice $\alpha=l$, corresponding to RSS with uncertainty ratio $A$ equal to that of the ground state, the RSS specialize from the three-parameter family of 
squeezed states to a two-parameter family of coherent states [40]. They then provide SQDT versions of the 'minimum-uncertainty coherent states' for three-dimensional systems that were introduced in Ref. [19]. However, they correspond to states of Rydberg atoms with high angular momentum. This means they are unsatisfactory as descriptions of Rydberg atoms prepared by excitation with a single short laser pulse with no external field present, since these have large $n$ and $l=1$. We therefore do not discuss these coherent states further in the present work.

\section{Properties of Radial Squeezed States}

In this section, we present some time-independent properties of the RSS. The time-dependent behavior is discussed in Sec. V.

First, we normalize $\psi(r)$. Imposing

$$
\int_{0}^{\infty} r^{2}|\psi(r)|^{2} d r=1
$$

gives

$$
N=\frac{\left(2 \gamma_{0}\right)^{2 \alpha+3}}{\Gamma(2 \alpha+3)}
$$

This normalization requires $\alpha \geq-1$ and $\gamma_{0}>0$.

Certain expectation values are of particular use in the description of the RSS and the comparison with other results. Here are a few key expectations, all calculated with normalized RSS. We find

$$
\begin{gathered}
\langle r\rangle=\frac{2 \alpha+3}{2 \gamma_{0}}, \quad\left\langle\frac{1}{r}\right\rangle=\frac{\gamma_{0}}{\alpha+1}, \\
\left\langle r^{2}\right\rangle=\frac{(\alpha+2)(2 \alpha+3)}{2 \gamma_{0}^{2}},\left\langle\frac{1}{r^{2}}\right\rangle=\frac{2 \gamma_{0}^{2}}{(\alpha+1)(2 \alpha+1)} \\
\left\langle p_{r}\right\rangle=-\gamma_{1} \quad, \quad\left\langle p_{r}{ }^{2}\right\rangle=\frac{\gamma_{0}^{2}}{2 \alpha+1}+\gamma_{1}^{2}
\end{gathered}
$$

It follows that the expectation value of the hamiltonian, which is the energy expectation value of the RSS, is given by

$$
\langle H\rangle=\frac{\gamma_{0}^{2}}{2(\alpha+1)(2 \alpha+1)}\left(2 \gamma_{0}^{2} l^{*}\left(l^{*}+1\right)+\alpha+1\right)-\frac{\gamma_{0}}{\alpha+1}+\frac{\gamma_{1}^{2}}{2} .
$$


From these expressions, it is apparent that the parameter $\alpha$ is further constrained by the requirement that the kinetic and potential energies of the RSS be separately normalizable, so that $\alpha>-\frac{1}{2}$.

It is also of interest to determine the uncertainties in the conventional coordinates $r$ and $p_{r}$. We find

$$
\Delta r=\frac{\sqrt{2 \alpha+3}}{2 \gamma_{0}}, \quad \Delta p_{r}=\frac{\gamma_{0}}{\sqrt{2 \alpha+1}} .
$$

This implies that the RSS obey the uncertainty relation

$$
\Delta r \Delta p_{r}=\frac{1}{2} \sqrt{\frac{2 \alpha+3}{2 \alpha+1}} .
$$

As might be expected, the RSS are not minimum-uncertainty states in the usual variables $r$ and $p_{r}$, although by construction they satisfy the equality in the minimumuncertainty relation (38).

The possible value of the uncertainty product ranges from an arbitrarily large value when $\alpha$ is near its minimum of $-\frac{1}{2}$, through $\Delta r \Delta p_{r}=\sqrt{3} / 2 \simeq 0.866$ when $\alpha=0$, to values falling as $\Delta r \Delta p_{r} \approx \frac{1}{2}(1+1 / \alpha)$ for large $\alpha$. As is discussed in Sec. V, large values of $\alpha$ are required to match key features of the RSS with the experimentally produced Rydberg wave packets at the first pass through the outer apsidal point of the orbit. We therefore expect the appropriate RSS to have uncertainty product close to $\frac{1}{2}$ at that time. This feature agrees with the behavior of Rydberg atoms excited by a short laser pulse. Numerical calculations performed for hydrogen show that $\Delta r \Delta p_{r}$ is close to its minimum when the electron is near the outer turning point [1, 2].

The radial probability distribution,

$$
f(r) \equiv r^{2}|\psi(r)|^{2}=N^{2} r^{2 \alpha+2} e^{-2 \gamma_{o} r}
$$

determines the shape of the RSS. The function $f(r)$ is asymmetrical in $r$. Its central maximum is located at $r=r_{0}$, where

$$
r_{0}=\frac{\alpha+1}{\gamma_{0}}=\frac{1}{\left\langle\frac{1}{r}\right\rangle} \text {. }
$$

The curvature of the envelope at $r_{0}$ can conveniently be measured by the ratio

$$
C_{0}=\frac{f^{\prime \prime}\left(r_{0}\right)}{f\left(r_{0}\right)}=-\frac{2 \gamma_{0}^{2}}{\alpha+1} .
$$


The specification of the RSS distribution is complete if $r_{0}$ and $C_{0}$ are known.

The RSS envelope $f(r)$ is a gamma distribution. The mean is just $\langle r\rangle$, while the variance is $(\Delta r)^{2}$. These are given in Eqs. (46) and (50). Other standard measures of the asymmetry of the function $f(r)$, such as the momental skewness or the kurtosis, can be obtained as simple expressions in terms of $\alpha$ and $\gamma_{0}$. The detailed shape of the distribution is determined by its moments $m_{k}$ about the mean, defined as

$$
m_{k}=\int_{0}^{\infty}(r-\langle r\rangle)^{k} f(r) r^{2} d r .
$$

These can be found by direct integration or as the coefficients of the power-series expansion of a relatively simple moment-generating function. In principle, the moments $m_{k}$ provide a basis for a detailed comparison of the RSS with wave packets obtained from other theory or experiment. We do not further pursue this line of inquiry here.

\section{TIME EVOLUTION OF RADIAL SQUEEZED STATES}

At this stage, we have identified the RSS as candidate Rydberg wave packets and have discussed several time-independent features. In this section, we discuss some time-dependent properties, in particular the RSS time evolution and autocorrelation functions. We also compare our results for the RSS with results presented elsewhere in the literature.

\section{A. Time Evolution: Theory}

Since the RSS have a relatively simple form, their behavior as a function of time can be studied both numerically and analytically. We have done both. Our numerical integrations are performed using the Crank-Nicholson method. Our analytical studies apply to the time evolution of RSS and to their autocorrelation functions, including issues such as characterizing full and fractional revivals as a function of the supersymmetry integer $I(l)$ and the quantum defect $\delta(l)$. This subsection presents some analysis for time-dependent properties. In what follows, we define the origin $t=0$ to be the time that the packet first reaches the outer apsidal point.

The full three-dimensional time-evolved wave packet can be written as a sum over 
the complete set of SQDT states:

$$
\Psi(\vec{r}, t)=Y_{10}(\theta, \phi) \sum_{n} c_{n} R_{n^{*} l^{*}}(r) e^{-i E_{n^{*} t}}
$$

where $l^{*}=1-\delta(1)+I(1)$ is specified for $\mathrm{p}$ states. In principle, the expansion includes the continuum states. However, for cases of interest the contributions from continuum states are negligible. The coefficients $c_{n}$ are determined by requiring that the initial wave function $\Psi(\vec{r}, 0)$ has radial piece given by the $\operatorname{RSS} \psi(r)$ of Eq. (43), i.e., $\Psi(\vec{r}, 0)=Y_{10}(\theta, \phi) \psi(r)$. This implies

$$
c_{n}=\left\langle R_{n^{*} l^{*}}(r) \mid \psi(r)\right\rangle
$$

Using the expression for $R_{n^{*} l^{*}}(r)$ in Eq. (四) and performing the integration, we get after some calculation the result

$$
\begin{aligned}
c_{n} & =N \frac{\Gamma\left(\alpha+l^{*}+3\right)}{\Gamma\left(l^{*}+1\right)}\left(\frac{\Gamma\left(n^{*}+l^{*}+1\right)}{\Gamma\left(n^{*}-l^{*}\right)}\right)^{\frac{1}{2}} \frac{n^{*(\alpha+1)}}{\left[n^{*}\left(\gamma_{0}+i \gamma_{1}\right)+1\right]^{\alpha+l^{*}+1}} \\
& \times{ }_{2} F_{1}\left(l^{*}+1-n^{*}, \alpha+l^{*}+3 ; 2 l^{*}+2 ; \frac{2}{n^{*}\left(\gamma_{0}+i \gamma_{1}\right)+1}\right) .
\end{aligned}
$$

Here, ${ }_{2} F_{1}$ is a hypergeometric function, and $N$ is the normalization constant given in Eq. (45).

The coefficients in Eq. (58) can also be found via a suitable integration over the analytical propagator for the Coulomb problem obtained recently in Ref. [41]. For the examples of interest, we find that the coefficients in the sum over states in Eq. (56) are strongly peaked around a central value $\bar{n}$ of $n$. Accurate numerical approximations can be obtained by truncating the sum to a subset of states centered on $\bar{n}$.

Another quantity of interest for the characterization of the time evolution is the absolute square of the autocorrelation function, $A(t)=|\langle\Psi(t) \mid \Psi(0)\rangle|^{2}$. In terms of the coefficients in Eq. (58), it is given as

$$
A(t)=\left.\left.\left|\sum_{n}\right| c_{n}\right|^{2} e^{-i E_{n^{*}} t}\right|^{2}
$$

Studies of the behavior of a hydrogenic radial packet as a function of time show that wavefunction dispersion is followed by the appearance of revivals [1, 11, 12]. We 
have repeated these analyses for the RSS within the context of the SQDT. As the RSS evolves in time from its initial configuration at $t=0$, it disperses. However, it is a trapped object, being bound to the atomic core. The dispersion therefore eventually leads to a situation in which what were initially the front and back parts of the packet interfere. This provides one signal for the eventual decoherence of the packet. For an RSS with eigenstate decomposition peaked about a principal quantum number $\bar{n}^{*}$ and dominantly contained within a range $\delta n^{*}$, we find that in the SQDT that the interference time $t_{\text {int }}^{*}$ at which the packet effectively collapses is given approximately by the expression

$$
t_{\mathrm{int}}^{*}=\frac{1}{3} \frac{\bar{n}^{*}}{\delta n^{*}} T_{\mathrm{cl}}^{*},
$$

where $T_{\mathrm{cl}}^{*}$ is given by Eq. (24).

At a time substantially later than $t_{\text {int }}$, the RSS reforms approximately into its original shape. For the SQDT, we find that this behavior occurs near a revival time $t_{\text {rev }}^{*}$ given by

$$
t_{\mathrm{rev}}^{*}=\frac{1}{3} \bar{n}^{*} T_{\mathrm{cl}}^{*} .
$$

The new wave packet oscillates with periodicity equal to the classical orbital period $T_{\mathrm{cl}}^{*}$. We refer to this packet as a full revival [42].

At certain times between $t_{\text {int }}^{*}$ and $t_{\text {rev }}^{*}$, the RSS gathers into $r$ spatially separated packets called fractional revivals. A full analysis in the SQDT, along the lines of that presented in Ref. [11], suggests that these times are given by rational fractions $2 p / q$ of the revival time $t_{\text {rev }}^{*}$, where $p$ and $q$ are relatively prime and where the number of packets is $r=q$ if $q$ is odd or $r=q / 2$ if $q$ is even. For the purposes of the discussions in the subsections below, we single out the particular fractional-revival times

$$
t_{r}^{*}=\frac{1}{r} t_{\mathrm{rev}}^{*} \quad .
$$

The wave-function period of these fractional revivals in the SQDT is given by

$$
T_{r}^{*}=\frac{1}{r} T_{\mathrm{cl}}^{*} .
$$


With our conventions, the full revival corresponds to the case $r=1$.

\section{B. Initialization}

To compare the properties of the RSS for different alkali-metal atoms with other theory and experiment, we need a procedure for determining the values of the parameters $\alpha, \gamma_{0}$, and $\gamma_{1}$ in a given situation. In a Rydberg atom that has been excited by a short laser pulse, the uncertainty product $\Delta r \Delta p_{r}$ is expected to be lowest at the first pass through the outer turning point [1, 2]. We therefore take this as our initialization point, and, as above, define it as the time origin $t=0$.

Fixing the initial form of the RSS requires the specification of three quantities. We choose these as the physical quantities $\left\langle p_{r}\right\rangle,\langle r\rangle$, and $\langle H\rangle$. The natural choice for the expectation value of the radial momentum at the apsidal point is zero. Similarly, a natural choice for the expectation value of $r$ at the initialization point is the outer apsidal point of the orbit,

$$
r_{\text {out }}^{*}=n^{* 2}\left[1+\sqrt{1-\frac{l^{*}\left(l^{*}+1\right)}{n^{* 2}}}\right] .
$$

Note that this differs from the outer apsidal point of the classical orbit, given in Eq. (23), by the use of the quantum-mechanical eigenvalue for the angular-momentum operator. Finally, one natural choice for the energy expectation value is the energy $E_{\bar{n}^{*}}=-1 / 2 \bar{n}^{* 2}$ of the central state $\bar{n}$ in the range of states excited by the short laser pulse.

Thus, we initialize the RSS by choosing the parameters $\alpha, \gamma_{0}, \gamma_{1}$ to satisfy the following equations:

$$
\begin{gathered}
\left\langle p_{r}\right\rangle=0 \\
\langle r\rangle=r_{\text {out }}^{*} \\
\langle H\rangle=E_{\bar{n}^{*}}
\end{gathered}
$$

The expectation values appearing in these equations are given in Eqs. (46), (48), and (49). The first condition, Eq. (65), can be inverted immediately to give $\gamma_{1}=0$. This 
leaves two conditions to fix the two remaining parameters $\alpha$ and $\gamma_{0}$ in terms of the two quantities $n^{*}$ and $l^{*}$. These in turn depend on the two quantities $I(l)$ and $\delta(l)$.

For the Rydberg wave packets obtained in experiment the value of $l^{*}$ is much smaller than the value of $n^{*}$. This means that the value of $\alpha$ is much greater than that of $\gamma_{0}$. In the cases we consider below, $\alpha$ is large and exceeds $\gamma_{0}^{2}$ by more than six orders of magnitude. From Eqs. (50) it follows that the initial squeezing in $p_{r}$ exceeds that in $r$ by about six orders of magnitude: $\Delta r / \Delta p_{r} \sim \alpha / \gamma_{0}^{2} \sim 10^{6}$. For more details about the uncertainty properties of the RSS, including a discussion of the time-dependence of the uncertainty product and ratio, see Ref. 24].

\section{Examples: Time Evolution}

In this subsection, we discuss two examples of the time evolution of RSS. The first is an RSS for hydrogen, with dominant energy component corresponding to principal quantum number $\bar{n}=85$. This example serves as a basis for comparison with other theoretical results. The second example is for rubidium. To determine the effects of the quantum defect on the time evolution, we choose for this case the same value of $\bar{n}$, which corresponds to $\bar{n}^{*} \simeq 82.35$.

For our first example, the SQDT reduces to the usual Coulomb case for p states: $I(1)=\delta(1)=0, l^{*}=l=1$, and $\bar{n}^{*}=\bar{n}=85$. From the conditions (66) and (67), we determine that the RSS parameters are $\alpha \simeq 168.225$ and $\gamma_{0} \simeq 0.0117465$. This gives an initial uncertainty product of $\Delta r \Delta p_{r} \simeq 0.50148$. We find that initially the wave packet moves toward the inner turning point. The packet decreases in amplitude, broadens, and starts to oscillate as it approaches the origin. After the bounce, it regathers into a coherent packet as it approaches the outer turning point again. We find that the periodic motion of the RSS is consistent with the classical orbital period $T_{\mathrm{cl}}^{*}=T_{\mathrm{cl}}=2 \pi \bar{n}^{3} \simeq 93.3$ psec.

Figures 3 and 4 show the envelope of the RSS at different times during the first orbit or so. In Fig. 3, the envelope of the RSS at $t=0$ is centered about the outer turning point near $r_{\text {out }} \approx 2 \bar{n}^{2} \simeq 14450$ a.u. The direction of propagation is towards the origin. The envelopes at times $t \simeq 19.4$ and 38.7 psec are also shown. Fig. 4 
shows the RSS envelopes after reflection off the core, while approaching and passing through the outer turning point again. From left to right, the graphs are at times $t \simeq 58.1,77.4$ and 96.8 psec. The latter plot displays a decreased amplitude because the packet is a few picoseconds into its second orbit and is therefore moving towards the origin again.

The generic behavior we find is consistent with the results of our perturbative calculation shown in Fig. 1. However, the perturbative calculation starts to break down after about one orbit, and comparisons cannot be made for long times. Our results compare well with Fig. 1(b) of Ref. [1], which shows the Rydberg wave packet during the second part of its orbit, obtained by numerically integrating the timedependent Schrödinger equation for an electron in hydrogen subject to a short laser pulse. This comparison suggests that the RSS are good models for radial wave packets in hydrogen.

Our second example involves rubidium p states with $\bar{n}=85$, which have $\delta(1) \simeq$ $2.65, I(1)=3, l^{*} \simeq 1.35$, and $n^{*} \simeq 82.35$. These values determine the RSS parameters as $\alpha \simeq 162.91$ and $\gamma_{0} \simeq 0.0121233$, with an initial uncertainty product $\Delta r \Delta p_{r} \simeq$ 0.50153 .

Figures 5 and 6 show the time evolution of the envelope of this RSS for rubidium. The time intervals in Figs. 5 and 6 are the same as those used for hydrogen in Figs. 3 and 4. Some differences between the rubidium and hydrogen examples are apparent. These may largely be attributed to the quantum defect. For example, the outer apsidal point for rubidium is $r_{\text {out }} \approx 2 \bar{n}^{* 2} \simeq 13560$ a.u. and is closer to the core than the corresponding point for hydrogen. Also, comparison of Figs. 3 and 5 shows that at $t \simeq 38.7 \mathrm{psec}$ the rubidium RSS is oscillating significantly more than the hydrogen one. This is a consequence of the reduction of the classical orbital period of rubidium, $T_{\mathrm{cl}}^{*}=2 \pi \bar{n}^{* 3} \simeq 84.9 \mathrm{psec}$, relative to that of hydrogen. At $38.7 \mathrm{psec}$, the rubidium RSS is closer to halfway through its orbit and hence exhibits more quantum-like behavior.

\section{Examples: Autocorrelation Functions}

In this subsection, we provide some examples of autocorrelation functions for 
RSS. Three cases are considered. The first two use the same RSS for hydrogen and rubidium considered in the previous subsection. The third example we use is an RSS for potassium with $\bar{n}=67$, which is convenient for comparison with experiment.

Our first example is the RSS for hydrogen with $\bar{n}=85$. The associated parameters $\alpha$ and $\gamma_{0}$ are given in the previous subsection. The discussion in Sec. V A suggests that the qualitative features of the motion are governed by the classical orbital period $T_{\mathrm{cl}} \simeq 93.3 \mathrm{psec}$, by the interference time $t_{\mathrm{int}} \simeq 4 T_{\mathrm{cl}}$ obtained with a spread $\delta n \sim 7$, and by the revival time $t_{\text {rev }} \simeq 2.6$ nsec.

Figure 7 shows the absolute square of the autocorrelation function for hydrogen with $\bar{n}=85$. Near $t=0$ the autocorrelation exhibits peaks spaced by the classical orbital period, reflecting the oscillatory motion between the outer and inner apsidal points expected at early times. About four orbits can be distinguished before the packet collapses. At times near the predicted value of $t_{\text {rev }}$, peaks spaced by the classical orbital period reappear. The figure also reveals the appearance of fractional revivals corresponding to the values $r=4,3$, and 2 at times $t_{4} \simeq 0.65 \mathrm{nsec}, t_{3} \simeq 0.88$ nsec, and $t_{2} \simeq 1.3 \mathrm{nsec}$, in agreement with the theory. As expected, these fractional revivals exhibit wave-function periodicities of $T_{4} \simeq 23.3 \mathrm{psec}, T_{3} \simeq 31.1 \mathrm{psec}$, and $T_{2} \simeq 46.7$ psec, respectively. Note that the maximum values of $A$ near the times $t=t_{r}$ decrease with increasing $r$, as might be expected from the spatial separations and numbers of the fractional revivals.

Our next example is the RSS for rubidium with $\bar{n}=85$. As described in the previous subsection, this corresponds to $\bar{n}^{*} \simeq 82.35$. The SQDT predicts a modified behavior of this RSS, with key features determined by the classical orbital period $T_{\mathrm{cl}}^{*} \simeq 84.9 \mathrm{psec}$, by the interference time $t_{\mathrm{int}}^{*} \simeq 4 T_{\mathrm{cl}}^{*}$, and by the revival time $t_{\mathrm{rev}}^{*} \simeq 2.3$ nsec. Note that although the interference time is different from that in hydrogen, it remains given by about four classical orbital periods because the values of $\bar{n}$ and $\delta n$ do not change significantly; cf. Eq. (60).

Figure 8 shows the absolute square of the autocorrelation function for this example. The overall behavior is similar to that of hydrogen. In agreement with the predicted interference time, about four orbits occur before wave-packet collapse. Larger correlations separated by the classical orbital period reappear near the theoretical 
value of $t_{\text {rev }}^{*}$. Fractional revivals with $r=4,3$, and 2 can be seen near $t_{4}^{*} \simeq 0.58$ nsec, $t_{3}^{*} \simeq 0.78 \mathrm{nsec}$, and $t_{2}^{*} \simeq 1.2 \mathrm{nsec}$, as expected. The associated periodicities are $T_{4}^{*} \simeq 21.2 \mathrm{psec}, T_{3}^{*} \simeq 28.3 \mathrm{psec}$, and $T_{2}^{*} \simeq 42.5$ psec.

Our last example is for the RSS in potassium with $\bar{n}=67$. The associated $\mathrm{p}$ states have $\delta(1) \simeq 1.71, I(1)=2, l^{*} \simeq 1.29$, and $n^{*} \simeq 65.29$. The corresponding RSS parameters are $\alpha \simeq 120.306$ and $\gamma_{0} \simeq 0.0142822$. The initial uncertainty product is $\Delta r \Delta p_{r} \simeq 0.50207$. For this case, the classical orbital period is $T_{\mathrm{cl}}^{*} \simeq 42.3$ psec. With $\delta n^{*} \sim 7$ still, the interference time is reduced to $t_{\mathrm{int}}^{*} \simeq 3 T_{\mathrm{cl}}^{*}$, i.e., about three orbits are expected to occur before the packet collapses. The revival time is now significantly smaller, $t_{\mathrm{rev}}^{*} \simeq 0.92$ nsec.

The absolute square of the autocorrelation function for this potassium RSS is shown in Fig. 9. Once again, the features agree with the theory. Only the fractional revivals with $r=3$ and 2 can be clearly seen, however. They occur near $t_{3}^{*} \simeq 0.31$ nsec and $t_{2}^{*} \simeq 0.46$ nsec, as predicted. The wave-function periodicities are $T_{3}^{*} \simeq 14.1$ psec and $T_{2}^{*} \simeq 21.2$ psec, respectively.

This example can be compared to the experiment presented in Ref. [7], in which potassium radial wave packets were excited in a pump-probe experiment. Oscillations in the photoion signal were observed that are consistent with $T_{\mathrm{cl}}^{*}$ and $t_{\mathrm{rev}}^{*}$ calculated from SQDT for a potassium RSS with $\bar{n}^{*} \simeq 65$. The revival peaks observed would correspond to the autocorrelation peaks exhibited in Fig. 9 starting at about 0.8 nsec. Similarly, examination of the experimental results presented in Refs. [8, 9] concerning the observation of fractional revivals with $r=2$ reveals behavior consistent with the analysis above.

\section{SUMMARY AND CONCLUSIONS}

In this paper, we have investigated two possibilites for describing Rydberg wave packets obtained by atomic excitation with short laser pulses. The approaches are somewhat complementary and both are developed in the context of SQDT, which can be viewed as an analytical model for single-body effective wave functions of Rydberg atoms. As such, the results we obtain are relevant both for hydrogen and 
for non-hydrogenic systems, including in particular the alkali-metal atoms used in experiments.

The first approach is a direct extension of time-dependent perturbation theory for the generation and evolution of the wave packets. The success of this approach suggests that the SQDT may be of use in calculations of this type carried out for alkali-metal atoms.

We have concentrated primarily on the second approach, which is an attempt to describe Rydberg wave packets in terms of a class of analytical squeezed states, the RSS. Using the SQDT, we succeeded in constructing the RSS as a three-parameter family of analytical states minimizing an uncertainty relation in nonstandard coordinates. The analytical form of the RSS, which are gamma distributions, means that relatively simple and/or analytical expressions can be obtained for various properties. The use of SQDT ensures that the RSS provide candidates for the analytical description of Rydberg wave packets in atoms other than hydrogen.

In terms of the radial coordinates $r$ and $p_{r}$, the RSS have close to minimum uncertainty for the cases we consider. To match with other theory and experiment in specific cases, we initialize the RSS at the outer apsidal point of the orbit. The three parameters are fixed by matching the expectation values of the position, momentum, and energy at that point. The ensuing behavior is completely determined. We derive relatively simple expressions for the time-evolved RSS and for their autocorrelation functions. We also predict the dependence of characteristic features of the behavior on the quantum defects. These include the initial orbital period, the time of packet collapse, and the full and fractional revival times and their associated orbital periodicities.

We have presented examples of time evolutions and autocorrelation functions both for hydrogen and for alkali-metal atoms. The behavior agrees with our theoretical expectations. The properties and behavior of the RSS are also in agreement with results on radial probability distributions, time evolution, and autocorrelation functions obtained by other authors for Rydberg wave packets. It appears that the RSS provide an analytical tool for describing and distinguishing experimental and theoretical features of Rydberg-atom radial wave packets produced by short-pulsed laser 
fields. 


\section{ACKNOWLEDGMENTS}

We enjoyed conversations with Charlie Conover and Duncan Tate. R.B. thanks Colby College for a Science Division Grant. Part of this work was performed while V.A.K. was visiting the Aspen Center for Physics.

\section{REFERENCES}

1. J. Parker and C.R. Stroud, Phys. Rev. Lett. 56, 716 (1986).

2. J. Parker and C.R. Stroud, Phys. Scr. T12, 70 (1986).

3. G. Alber, H. Ritsch, and P. Zoller, Phys. Rev. A 34, 1058 (1986).

4. G. Alber and P. Zoller, Phys. Rep. 199, 231 (1991).

5. A. ten Wolde, L.D. Noordam, A. Lagendijk, and H.B. van Linden van den Heuvell, Phys. Rev. Lett. 61, 2099 (1988).

6. J.A. Yeazell, M. Mallalieu, J. Parker, and C.R. Stroud, Phys. Rev. A 40, 5040 (1989).

7. J.A. Yeazell, M. Mallalieu, and C.R. Stroud, Phys. Rev. Lett. 64, 2007 (1990).

8. J.A. Yeazell and C.R. Stroud, Phys. Rev. A 43, 5153 (1991).

9. D.R. Meacher, P.E. Meyler, I.G. Hughes, and P. Ewart, J. Phys. B 24, L63 (1991).

10. A. ten Wolde, L.D. Noordam, H.G. Muller, and H.B. van Linden van den Heuvell, in F. Ehlotzky, ed., Fundamentals of Laser Interactions II (SpringerVerlag, Berlin, 1989).

11. I.Sh. Averbukh and N.F. Perelman, Phys. Lett. 139A, 449 (1989).

12. M. Nauenberg, J. Phys. B 23, L385 (1990).

13. J. Grochalicki and M. Lewenstein, J. Phys. B 21, 3285 (1988).

14. L.D. Noordam, D.I. Duncan, and T.F. Gallagher, Phys. Rev. A 45, 4734 (1992).

15. See, for example, J.R. Klauder and B.-S. Skagerstam, eds., Coherent States (World Scientific, Singapore, 1985); W.-M. Zhang, D.H. Feng, and R. Gilmore, 
Rev. Mod. Phys. 62, 867 (1990).

16. E. Schrödinger, Naturwissenschaften 14, 664 (1926).

17. L.S. Brown, Am. J. Phys. 41, 525 (1973).

18. J. Mostowski, Lett. Math. Phys. 2, 1 (1977).

19. M.M. Nieto, Phys. Rev. D 22, 391 (1980); V.P. Gutschick and M.M. Nieto, Phys. Rev. D 22, 403 (1980).

20. D.S. McAnally and A.J. Bracken, J. Phys. A 23, 2027 (1990).

21. M. Nauenberg, Phys. Rev. A 40, 1133 (1989).

22. J.-C. Gay, D. Delande, and A. Bommier, Phys. Rev. A 39, 6587 (1989).

23. Z. Dačić Gaeta and C.R. Stroud, Phys. Rev. A 42, 6308 (1990).

24. R. Bluhm and V.A. Kostelecký, Phys. Rev. A 48, R4047 (1993) (quantph/9508019).

25. V. A. Kostelecký and M. M. Nieto, Phys. Rev. Lett. 53, 2285 (1984); Phys. Rev. A 32, 1293 (1985).

26. V. A. Kostelecký and M. M. Nieto, Phys. Rev. A 32, 3243 (1985).

27. H. Nicolai, J. Phys. A 9, 1497 (1976); E. Witten, Nucl. Phys. B185, 513 (1981).

28. J. R. Rydberg, Kongl. Sven. vetensk.-akad. hand. 23, no. 11 (1890); Philos. Mag. 29, 331 (1890).

29. See, for example, R.D. Cowan, The Theory of Atomic Structure and Spectra (University of California, Berkeley, 1981).

30. C.H. Greene, U. Fano, and G. Strinati, Phys. Rev. A 19, 1485 (1979); C.H. Greene, A. Rau, and G. Strinati, Phys. Rev. A 26, 2441 (1982); M.J. Seaton, Rep. Prog. Phys. 46, 167 (1983).

31. See also G. Simons, J. Chem. Phys. 60, 645 (1974); V. A. Kostelecký and M. M. Nieto, Phys. Rev. A 43, 4063 (1991) and references therein.

32. M.T. Djerad, J. Phys. II 1, 1 (1991).

33. R. Bluhm and V.A. Kostelecký, Phys. Rev. A 47, 794 (1993).

34. M.L. Zimmerman, M.G. Littman, M.M. Kash, and D. Kleppner, Phys. Rev. A 
20, 2251 (1979); D. Kleppner, M.G. Littman, and M.L. Zimmerman, in R.F Stebbings and F.B. Dunning, eds., Rydberg States of Atoms and Molecules (Cambridge University Press, Cambridge, 1983), p. 73.

35. T.F. Gallagher, in H.J. Beyer and H. Kleinpoppen, eds., Progress in Atomic Spectroscopy, Part D (Plenum, New York, 1987).

36. V. A. Kostelecký, M. M. Nieto, and D. R. Truax, Phys. Rev. A 38, 4413 (1988).

37. V.A. Kostelecký, in B. Gruber and T. Otsuka, eds., Symmetries in Science VII: Spectrum Generating Algebras and Dynamic Symmetries in Physics (Plenum, New York, 1993) (quant-ph/9508015).

38. V.A. Kostelecký, in D. Han, Y.S. Kim, and W.W. Zachary, eds., Proceedings of the Workshop on Harmonic Oscillators (NASA, Washington, D.C., 1993), p. 443; Supersymmetry, Coherent States, and Squeezed States for Rydberg Atoms, in D.H. Feng, J.R. Klauder, and M.R. Strayer, eds., Proceedings of the International Symposium on Coherent States: Past, Present, and Future, World Scientific, Singapore, 1994.

39. See, for example, M.M. Nieto, in G.T. Moore and M.O. Scully, eds., Frontiers of Nonequilibrium Statistical Physics (Plenum, New York, 1986), p. 287.

40. We remark that similar squeezed states for the pure Coulomb problem were obtained as part of the method of derivation of the 'minimum-uncertainty coherent states' in Ref. [19]. Although not discussed as such in those papers, the role of these objects as squeezed states was subsequently pointed out in Ref. [39].

41. S.M. Blinder, Phys. Rev. A 43, 13 (1991).

42. The reader is warned that for the hydrogenic case some difference in nomenclature exists in the literature, with this new packet often called a 'one-half revival.' The full revival time then may be taken as the quantity equivalent to $2 t_{\mathrm{rev}}^{*}$, although evidently with zero supersymmetry integer $I(l)$ and zero quantum defect $\delta(l)$. 


\section{Figure Captions}

Fig. 1: Hydrogen radial wave packet with $\bar{n}=85$. The unnormalized radial probability distribution $f(r)$ is plotted as a function of radial distance $r$ in a.u. From left to right, the graphs are shown for the times $t=\frac{1}{9} T_{\mathrm{cl}}, \frac{2}{9} T_{\mathrm{cl}}, \frac{3}{9} T_{\mathrm{cl}}$, and $\frac{4}{9} T_{\mathrm{cl}}$, where $T_{\mathrm{cl}}=93.3$ psec.

Fig. 2: Rubidium radial wave packet with $\bar{n}=85$. The unnormalized radial probability distribution $f(r)$ is plotted as a function of radial distance $r$ in a.u. From left to right, the graphs are shown at the same times as in Fig. 1: $t=10.4$, $20.7,31.1$, and 41.5 psec.

Fig. 3: Hydrogen RSS with $\bar{n}=85$ : first part of the orbital motion. The unnormalized radial probability distribution $f(r)$ is plotted as a function of radial distance $r$ in a.u. From right to left, the graphs are shown at the times $t=0$, 19.4, and 38.7 psec.

Fig. 4: Hydrogen RSS with $\bar{n}=85$ : second part of the orbital motion. The unnormalized radial probability distribution $f(r)$ is plotted as a function of radial distance $r$ in a.u. From left to right, the graphs are shown at the times $t=58.1$, 77.4 , and 96.8 psec.

Fig. 5: Rubidium RSS with $\bar{n}=85$ : first part of the orbital motion. The unnormalized radial probability distribution $f(r)$ is plotted as a function of radial distance $r$ in a.u. From right to left, the graphs are shown at the same times as in Fig. 3.

Fig. 6: Rubidium RSS with $\bar{n}=85$ : second part of the orbital motion. The unnormalized radial probability distribution $f(r)$ is plotted as a function of radial distance $r$ in a.u. From left to right, the graphs are shown at the same times as in Fig. 4.

Fig. 7: Absolute square of the autocorrelation function for hydrogen RSS with $\bar{n}=$ 85 as a function of time in nanoseconds.

Fig. 8: Absolute square of the autocorrelation function for rubidium RSS with $\bar{n}=$ 85 as a function of time in nanoseconds.

Fig. 9: Absolute square of the autocorrelation function for potassium RSS with $\bar{n}=$ 67 as a function of time in nanoseconds. 
This figure "fig1-1.png" is available in "png" format from: http://arxiv.org/ps/quant-ph/9508020v1 
This figure "fig2-1.png" is available in "png" format from: http://arxiv.org/ps/quant-ph/9508020v1 
This figure "fig3-1.png" is available in "png" format from: http://arxiv.org/ps/quant-ph/9508020v1 
This figure "fig1-2.png" is available in "png" format from: http://arxiv.org/ps/quant-ph/9508020v1 
This figure "fig2-2.png" is available in "png" format from: http://arxiv.org/ps/quant-ph/9508020v1 
This figure "fig3-2.png" is available in "png" format from: http://arxiv.org/ps/quant-ph/9508020v1 
This figure "fig1-3.png" is available in "png" format from: http://arxiv.org/ps/quant-ph/9508020v1 
This figure "fig2-3.png" is available in "png" format from: http://arxiv.org/ps/quant-ph/9508020v1 
This figure "fig3-3.png" is available in "png" format from: http://arxiv.org/ps/quant-ph/9508020v1 